\title{
Radical stabilization of aromatic diacetylenes (dinaphthylbutadiynes) in the free radical polymerization of methyl methacrylate
}

\author{
Miriam F Beristain ${ }^{1,2}$, Mirna R Estrada ${ }^{1}$, Alejandra Ortega ${ }^{1}$, Angel Licea Claverie ${ }^{3}$ and Takeshi Ogawa ${ }^{2}$
}

Polymer Journal (2016) 48, 963-967; doi:10.1038/pj.2016.54; published online 8 June 2016

\section{INTRODUCTION}

The free radical polymerization, particularly living radical polymerization, of vinyl monomers has been extensively investigated to develop polymers with desired molecular weights and distributions. In these studies, ESR spectroscopy is an important tool to study the nature of propagating radicals; however, direct observation of the short-lived transient propagating radicals remains difficult unless the polymerization system is frozen or become viscous, rendering the motion of propagating radicals slow or immobile. Indirect examination of ESR spectra using spin-trapping agents such as nitrones or nitroso compounds, ${ }^{1}$ nitroxide ${ }^{2}$ and dithio compounds ${ }^{3}$ that form stable radicals is possible. These trapping agents can be detected by ESR spectroscopy and undergo fragmentation to regenerate propagating radicals. Kajiwara ${ }^{4}$ recently reported a detailed investigation on ESR detection of propagating radicals during the photopolymerization of acrylates and methacrylates with bulky groups. A few other cases in which ESR spectra of propagating radicals are observed during polymerization include the polymerization of methyl methacrylate (MMA) in the presence of lithium triflate, in which the lithium cation interacts with the oxygen atoms of MMA, stabilizing the propagating polymethylmethacrylate (PMMA) radicals and thus increasing the radical concentration. ${ }^{5}$ The electrostatic repulsion between the complexed propagating radicals decreases the rate of termination. Previously, we studied the effects of diphenylbutadiyne (DPB) on the free radical polymerization of various vinyl monomers and found that the rate of polymerization significantly decreased in the presence of diphenylbutadienes, ${ }^{6}$ and electron spin resonance (ESR) signals of propagating radicals such as polymethacrylates and polystyrene radicals were observed during polymerization. ${ }^{7,8}$ In the case of the ethyl acrylate, the polymerization was inhibited by DPB but the ESR signal of polyethylacrylate radicals was observed even at $100^{\circ} \mathrm{C}$ when the system was made viscous. ${ }^{9}$ Interestingly, no fragment of aromatic diacetylenes (ADAs) was found in the polymers obtained, indicating that the diacetylenes do not form bonds with the propagating radicals. Therefore, these ADAs are not chain transfer agents but are considered free radical stabilizers, as shown in Scheme 1. Here, the interacted form (I) of the radicals, which is influenced by the electronic density of ADAs, was detected by the ESR. For example, in the case of DPB and di (methoxycarbonyl) diphenylbutadiyne, the rate and degree of polymerization of MMA decreased and strong ESR signals were observed during polymerization, which did not quench indefinitely under inert atmosphere. In the case of ADAs with donor groups, such as dimethoxydiphenylbutadiyne or diaminodiphenylbutadiyne, the decrease in the rate was not appreciable, and the ESR signals were weaker and slowly disappeared when the polymerization was stopped. ${ }^{8}$ Interaction of ADAs with free radicals was also evidenced by the retardation of the gamma ray degradation of commercial polyurethane in the presence of a small amount of DPB. ${ }^{10}$ These results suggest that ADAs are a new type of free radical stabilizer or electron acceptor. In the present study, two naphthylbutadiynes, 1,4-dinaphthyl-1,3-butadiyne (DNB) and $4,4^{\prime}$-dinitrodinaphthylbutadiyne were synthesized, and their effects on the free radical polymerization of MMA were investigated to obtain more information on the interaction of ADAs in free radical reactions.

\section{EXPERIMENTAL PROCEDURE}

\section{Materials}

All reagents were purchased from Aldrich Chemical (St Louis, MO, USA) and used without further purification unless otherwise noted. MMA was distilled at reduced pressure and azobisisobutyronitrile was recrystallized from ethanol. Benzene was supplied by Fermont and was washed with sulfuric acid and sodium hydroxide solution before distillation. The chemical structures of ADAs mentioned in this study are shown in Scheme 2.

\section{Synthesis of dinaphthylbutadiynes}

1,4-Dinaphthyl-1,3-butadiyne (DNB), a known diacetylene, ${ }^{11,12}$ was prepared from the oxidative coupling of 1-ethynylnaphthalene in acetone at room temperature. Recrystallization from toluene and activated charcoal afforded the desired compound as reddish-orange, shiny crystals (m.p. $=174-176^{\circ} \mathrm{C}$ ).

For the synthesis of DNNB (Scheme 3), 1-bromo-4-nitronaphthalene (BNN) was prepared according to the method reported by Jones. ${ }^{13}$ Recrystallization

${ }^{1}$ Instituto de Investigaciones en Materiales, Universidad Nacional Autónoma de México, Circuito Exterior, Ciudad Universitaria, México City, México; ${ }^{2}$ Centro de Nanociencias y Nanotecnología, Universidad Nacional Autónoma de México, Ensenada, México and ${ }^{3}$ Centro de Graduados e Investigacion, Instituto Tecnologico de Tijuana, Tijuana, México Correspondence: Professor T Ogawa, Centro de Nanociencias y Nanotecnología, Universidad Nacional Autónoma de México, Km 107 Hwy Tijuana-Ensenada, Ensenada, BC 22860, México.

E-mail: ogawa@unam.mx

Received 19 November 2015; revised 6 April 2016; accepted 6 April 2016; published online 8 June 2016 
<smiles>[R]c1ccc(C#CC#Cc2ccc(C#CC#Cc3ccc([R])cc3)cc2)cc1</smiles>

Scheme 1 Stabilization of propagating PMMA radicals by aromatic diacetylenes.


$\mathrm{COOCH}_{3}$ (dimethoxycarbonyldiphenylbutadiyne)

Scheme 2 Chemical structures of aromatic diacetylenes.<smiles>O=[N+]([O-])c1ccc(Br)c2ccccc12</smiles>

BNN

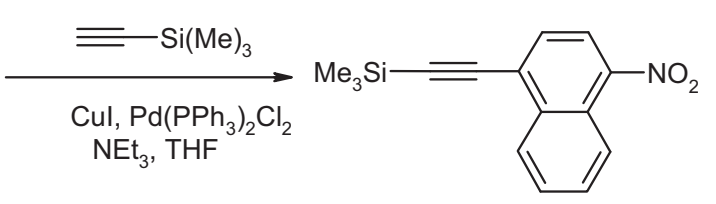

$\mathrm{K}_{2} \mathrm{CO}_{3}$ Acetone

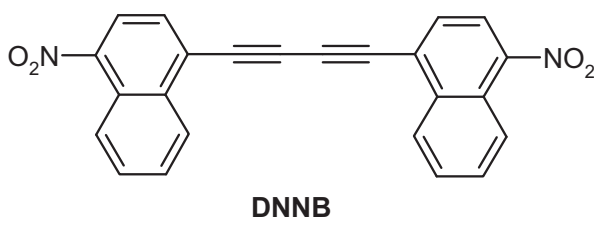

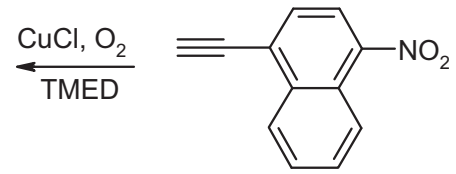

Scheme 3 Synthetic route of 1,4'-di(4-nitronaphthyl)-1,3-butadiyne (DNNB).

from ethanol afforded a yellow powder (m.p. $=68-70^{\circ} \mathrm{C}$ ). BNN was reacted with trimethylsilylacetylene using bistriphenylphosphine palladium (II) dichloride and copper (I) iodide in tetrahydrofuran and triethylamine at room temperature under nitrogen atmosphere. The solution was filtered, and the solvent was evaporated under reduced pressure. The remaining solid was washed with diluted hydrochloric acid, followed by a mixture of hexane: ethyl acetate (95:5) to yield the desired compound as a yellow powder $(78 \%$, m.p. $=78-80^{\circ} \mathrm{C}$ ). The deprotection of the acetylene was accomplished in acetone with potassium carbonate at room temperature under nitrogen atmosphere for $8 \mathrm{~h}$. After this time, the solvent was removed under reduced pressure and the remaining solid was dissolved in dichloromethane to remove insoluble material. The terminal acetylene was recovered after solvent evaporation, and it was used for the oxidative coupling reaction without further purification. The oxidative coupling reaction was carried out using copper (I) chloride and $N, N, N^{\prime} N^{\prime}$ tetramethylethylenediamine in acetone in the presence of gently bubbling oxygen. After $48 \mathrm{~h}$, the solvent was evaporated, and the product was washed with ammonium hydroxide solution to remove the copper, followed by washing with acetone, ethanol and ethyl acetate to afford 1,4'-di(4-nitronaphthyl)-1,3-butadiyne (DNNB) as a yellow solid $\left(40 \%\right.$, m.p. $\left.=223^{\circ} \mathrm{C}\right)$. UV - vis tetrahydrofuran (THF): $\lambda_{\max }=390 \mathrm{~nm}$. ${ }^{1} \mathrm{H}-\mathrm{NMR}\left(400 \mathrm{MHz}\right.$, DMF- $\left.d_{6}\right) \delta: 7.96(\mathrm{~d}, 2 \mathrm{H}), 8.21(\mathrm{~d}, 1 \mathrm{H}), 8.39$ (d, $\left.1 \mathrm{H}\right), 8.46$ $(\mathrm{s}, 1 \mathrm{H}), 8.56(\mathrm{~s}, 1 \mathrm{H})$. 


\section{Polymerization}

The polymerization of MMA was carried out in a three-necked flask, in which were placed $20 \mathrm{ml}(0.187 \mathrm{~mol})$ of MMA, $0.1312 \mathrm{~g}(0.0008 \mathrm{~mol})$ of azobisisobutyronitrile dissolved in benzene $(20 \mathrm{ml})$ and $0.0004 \mathrm{~mol}$ of additives (DNB, DNNB and BNN) dissolved in dimethylformamide (DMF) $(5 \mathrm{ml})$ (total volume $=45 \mathrm{ml} ;[\mathrm{MMA}]=4.16 \mathrm{M} ;[\mathrm{AIBN}]=0.018 \mathrm{M} ;[\mathrm{ADAs}]=0.009 \mathrm{M})$. The polymerization solutions were degassed by freeze-thaw cycles, and the polymerization was carried out with stirring at $60^{\circ} \mathrm{C}$ under argon atmosphere. The polymerization was monitored by removing small aliquots at various time intervals, and the polymer was precipitated in methanol. The polymers thus obtained were purified by re-precipitation from their dichloromethane solution into methanol. For the polymer obtained in the presence of DNNB, the product was dissolved in benzene and precipitated in triethylamine. This process was repeated three times after which time the product was dissolved in dichloromethane and activated charcoal was added. The solution was filtered, and the polymer was precipitated in methanol. The low concentration of ADAs used in this work is due to poor solubility in MMA.

\section{Characterization}

${ }^{1} \mathrm{H}-\mathrm{NMR}$ spectra of the naphthylbutadiynes were recorded on a Bruker Avance $400 \mathrm{MHz}$ spectrometer (Bruker, Billerica, MA, USA). Fourier transform infrared (FT-IR) spectra were taken on a Thermo Scientific Nicolet 6700 FTIR spectrometer (ThermoScientific, Waltham, MA, USA). The UV-visible spectra of PMMA were taken using an Agilent Cary $60 \mathrm{UV}$ - vis spectrophotometer (Agilent, Santa Clara, CA, USA) on films prepared by spin-coating onto quartz substrates. The molecular weights were determined by gel permeation chromatography at $25^{\circ} \mathrm{C}$ in THF at a flow rate of $1 \mathrm{ml} \mathrm{min}^{-1}$, using a Viscotek Malvern 305 TDA chromatograph (Viscotek Malvern, Westborough, MA, USA) equipped with refractive index, viscosity and right-angle light-scattering detectors. Separation was performed on porous styrene-divinylbenzene copolymer-based columns with two analytical columns in series $300 \times 7.8 \mathrm{~mm}$ (CLM3009, T6000M; General Mixed Organic). Polystyrene standards were used to confirm the accuracy of the determination. A $\mathrm{dn} / \mathrm{dc}$ value of $0.086 \mathrm{mlg}^{-1}$ was used for molecular weight calculations. The ESR measurements were performed in a JEOL ESR spectrometer Model RE3X using quartz tubes with an inner diameter of $3 \mathrm{~mm}$, supplied by Wilmad-LabGlass (Vineland, NJ, USA). The measurements were performed under the following conditions: microwave frequency: $9.127 \mathrm{GHz}$, central field, H: $3300 \pm 400 \mathrm{G}$, modulation frequency: $100 \mathrm{kHz}$, modulation amplitude: $5 \mathrm{G}$, receiver gain: 400 for DNB and 200 for DNNB. ESR tubes were filled with aliquots taken from the polymerization solution. These aliquots were degassed
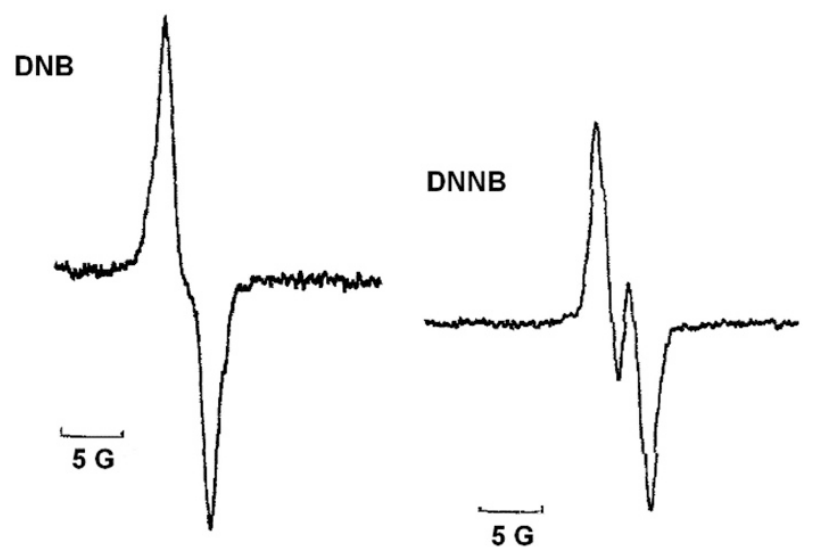

Figure 1 ESR spectra of PMMA radicals in the presence of DNB and 1,4'-di(4-nitronaphthyl)-1,3-butadiyne (DNNB) at $60^{\circ} \mathrm{C}$ after $195 \mathrm{~min}$. $[\mathrm{MMA}]=4.45 \mathrm{M} ; \quad[\mathrm{AlBN}]=0.019 \mathrm{M} ; \quad[\mathrm{DNB}]$ and $[\mathrm{DNNB}]=0.0095 \mathrm{~m}$. Microwave frequency: $9.127 \mathrm{GHz}$, central field, $\mathrm{H}: 3300 \pm 400 \mathrm{G}$, modulation frequency: $100 \mathrm{kHz}$, modulation amplitude: $5 \mathrm{G}$, receiver gain: 400 for DNB and 200 for DNNB. AIBN, azobisisobutyronitrile; MMA, methyl methacrylate. by a freeze-thaw process and then sealed off in vacuum. The tubes were heated at $60{ }^{\circ} \mathrm{C}$ in an oil bath for $95 \mathrm{~min}$ before ESR measurements. The concentration of free radicals was estimated by the double numerical integration of the first derivative of the resonance curve. ${ }^{14}$

\section{RESULTS AND DISCUSSION}

As shown in Figure 1, the ESR signals of the propagating PMMA radicals were observed during polymerization in the presence of naphthylbutadiynes, which is similar to the polymerization of MMA in the presence of $\mathrm{DPB} .^{7}$ In the case of DPB, the signals of PMMA radicals were not observed in the absence of DPB unless the polymerization system became very viscous or almost solid $\left(13.5 \mathrm{~h}\right.$ at $\left.54.7^{\circ} \mathrm{C}\right)$, whereas in the presence of $\mathrm{DPB}$, the signals appeared after $18 \mathrm{~min}$ of polymerization. The intensity of the ESR signals, which correspond to the PMMA-propagating radicals interacting with $\mathrm{ADAs}$, was higher for the polymerization system of DNNB than that of DNB (note: the receiver gain for DNNB is half of that for DNB). Figure 2 shows the increase in concentration of the ESR-detectable radicals over time. The concentration of the radicals in the case of DNNB is approximately double that of DNB. This implies that the electron-withdrawing nitro group of DNNB allows PMMA radicals to be more readily trapped (stabilized) than in the case of DNB. This trend was also observed for derivatives of DPB. ${ }^{8}$ The degree of the interaction was dependent on the substituent groups, and the reduction in the rate of polymerization and molecular weight was less prominent in DPB derivatives containing donor substituents such as amino and methoxy groups, than those with electron acceptor groups such as methoxycarbonyl. For example, in systems containing DPB derivatives with acceptor substituents, the ESR signals did not decay for weeks when removed from the spectrometer cavity. However, for systems containing DPBs with electron donors, the ESR signals disappeared in a few hours when removed from the cavity, indicating that the lower the electronic density of ADAs, the stronger the interaction.

The time-conversion curves at the initial stage of polymerization are shown in Figure 3. The polymerization rate decreased slightly in the presence of the DNNB due to the stabilization of PMMA radicals. Our previous work noted even larger decreases in the rates due to an approximately 30 times higher concentration of ADAs. ${ }^{6,8}$

Some naphthalenes, such as naphthols and naphthylamines that contain a labile hydrogen, are known to inhibit the polymerization of MMA. ${ }^{15}$ DNB and DNNB do not have labile hydrogens; nevertheless,

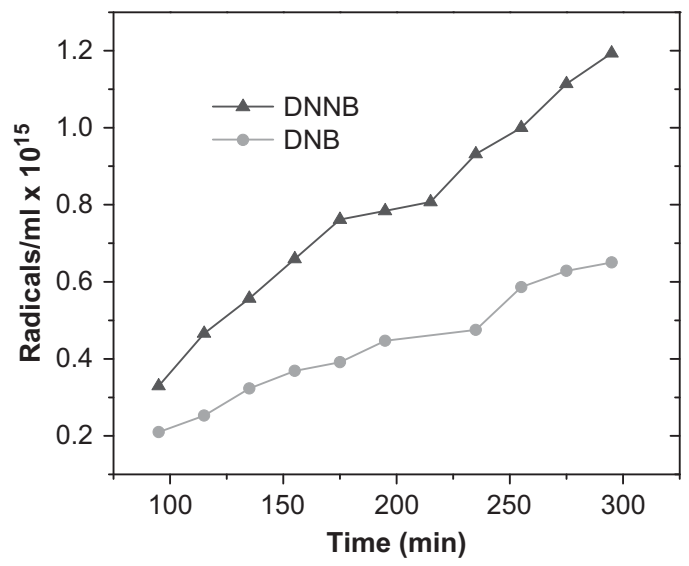

Figure 2 Changes in the radical concentration with polymerization time. A full color version of this figure is available at Polymer Journal online. 
the reaction was carried out in the presence of $\mathrm{BNN}$ to assess the influence of the naphthalene ring on the polymerization. As seen in Figure 3, no appreciable retardation took place with BNN.

The changes in the molecular weights of the polymers up to $25 \%$ conversion are shown in Figure 4. In all cases, the molecular weights

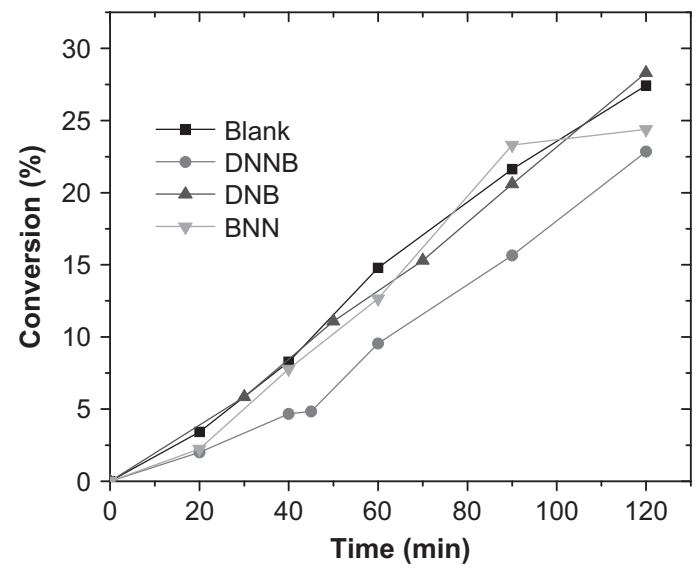

Figure 3 Time-conversion curves of the polymerization of methyl methacrylate. A full color version of this figure is available at Polymer Journal online.

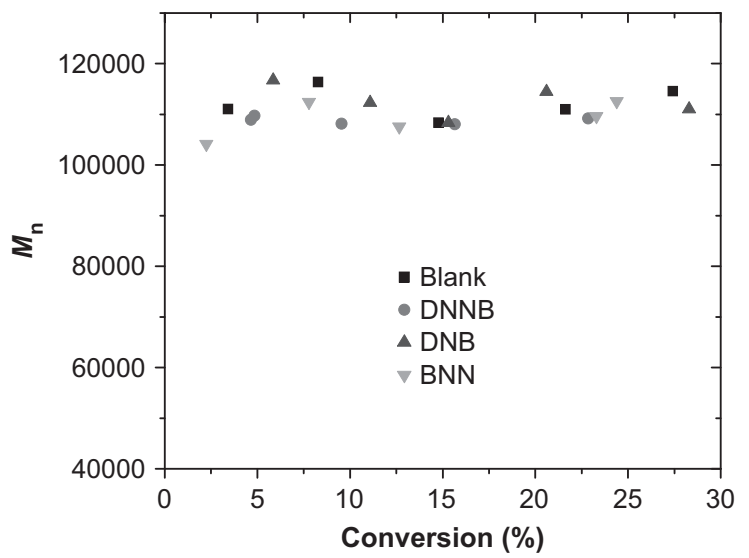

Figure 4 Relationships between conversion and number average molecular weight of PMMA. A full color version of this figure is available at Polymer Journal online.

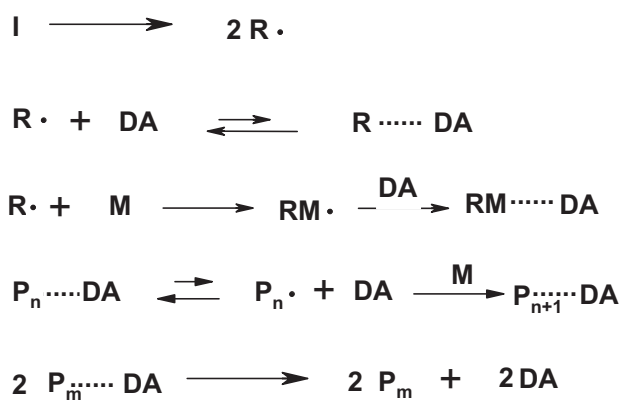

Scheme 4 Plausible polymerization mechanism in the presence of aromatic diacetylenes. DA, aromatic diacetylene; I, initiator; M, monomer; P, polymer; P.....DA, stabilized radical; $\mathrm{Pn} \bullet$, freed propagating radical; R•, primary radical from I. remain in the range of around 115000 , verifying that the effect of the ADAs is negligible. The polydispersity, which ranged from 1.6 to 2, was also not affected by the presence of the ADAs. Because molecular weights increase with conversion in the cases of living polymerization of MMA, ${ }^{16,17}$ it is clear that the polymerization in the presence of ADAs is not a controlled living polymerization.

A plausible mechanism of polymerization is shown in Scheme 4. At the initial stage of polymerization, the interaction of ADAs with R $\bullet$ exists (reaction 2); however, because of the small size of $\mathrm{R} \bullet$ and the low viscosity, the dissociation proceeds rapidly (reaction 2 ). Therefore, the initiation (reaction 3 ) is not affected by the presence of the ADAs. As the viscosity increases, the dissociation decreases, producing an increase in the concentration of ESR-detectable radicals. The propagation may take place by the addition of monomers to the stabilized radicals, but it is more likely that the stabilized radicals momentarily dissociate and add to the monomer, and then the newly formed radical is promptly restabilized (reaction 4).

$\mathrm{ADAs}$ are not incorporated into the polymer chains as confirmed by the FT-IR spectra (Figure 5) and the UV - visible absorption spectra (Figure 6) of the PMMA obtained in the presence ADAs. In particular, the UV-visible absorption of ADAs in the range of $300-450 \mathrm{~nm}$

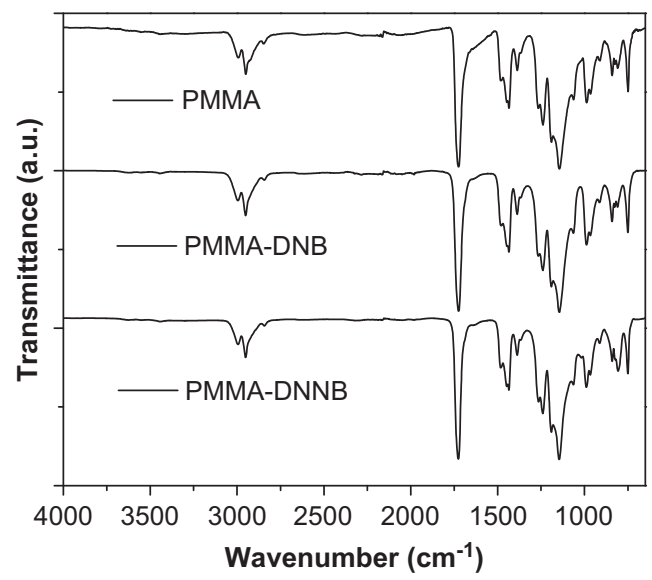

Figure 5 FT-IR spectra of PMMA.

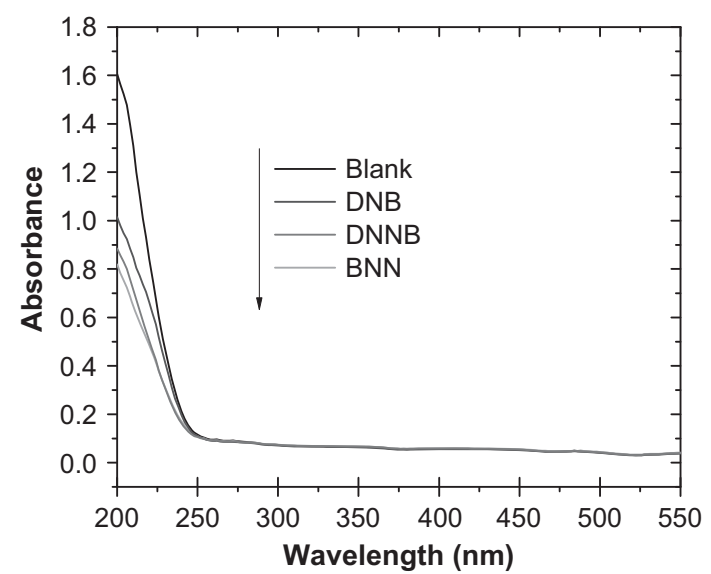

Figure 6 UV-visible absorption spectra of PMMA obtained in the absence and presence of DNB, 1,4'-di(4-nitronaphthyl)-1,3-butadiyne (DNNB) and 1-bromo-4-nitronaphthalene (BNN) spin-coated films. A full color version of this figure is available at Polymer Journal online. 


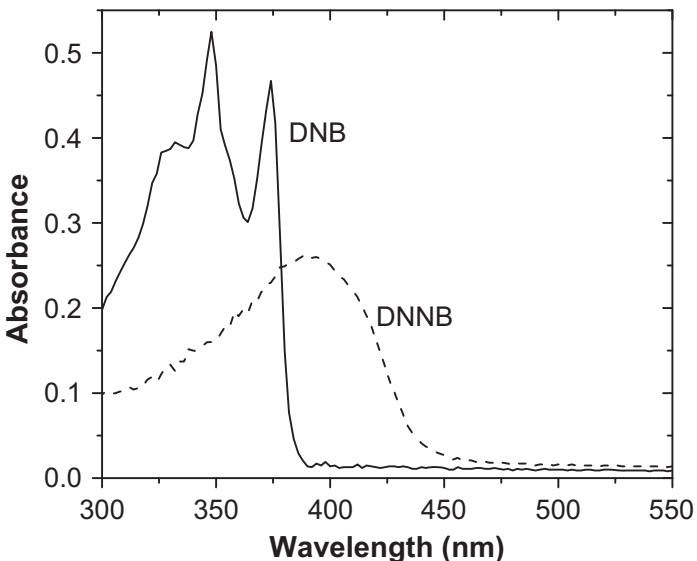

Figure 7 UV-visible absorption spectra of DNB and 1,4'-di(4-nitronaphthyl)-1,3-butadiyne (DNNB) in THF.

(Figure 7) are not observed in the PMMA spectra. Similar results were also observed for the polymerization of ethyl acrylate in the presence of DPB. ${ }^{9}$ Therefore, as summarized in Scheme 1, (I) is the ESR-detectable radical and the adduct (II) is not formed, implying that ADAs behave as electron acceptors, and this unique behavior is the result of external stabilization of radical species by ADAs, without the formation of a sigma bond with propagating radicals. Thus, diacetylenes do not suffer from the addition of vinyl propagating radicals under the normal polymerization conditions. This behavior is different from other electron acceptors, such as fullerenes and carbon nanotubes, ${ }^{18,19}$ in which the ESR signals observed in the polymerization of vinyl monomers ${ }^{20-22}$ correspond to the ESR signals of fullerene and nanotube radicals formed by the addition of propagating radicals.

\section{CONCLUSION}

DNB and DNNB stabilize PMMA radicals, allowing observation of the propagating radicals by ESR spectroscopy at the polymerization temperature. The radical concentration increases with conversion because of the increase in viscosity of the polymerization system, which likely decreases the dissociation of stabilized radicals. This dissociation depends on the electronic density of the ADAs-the stronger the electron-accepting character, the higher the ESRdetectable radicals. The rate of polymerization decreased slightly in the presence of DNNB, but no significant change was observed in the case of DNB. The poor solubility of DNNB in MMA prevented assessment of the steric effect of the naphthalene rings compared with DPB. The molecular weights did not increase with conversion, and the polydispersity was approximately 1.8 , indicating that the polymerization in the presence of ADAs is not a living polymerization. Unlike other radical stabilizers, ADAs are not incorporated into the polymer chains; therefore, they can be considered a new class of free radical stabilizers and electron acceptors.

\section{CONFLICT OF INTEREST}

The authors declare no conflict of interest.

\section{ACKNOWLEDGEMENTS}

This work was supported by a grant from CONACYT under the contract CB2011-165507. MFB expresses her gratitude to CONACYT (165507) for her fellowship. Thanks are also due to I Zapata and G Cedillo for their assistance in GPC and NMR spectroscopy, respectively.

1 Janzen, E. G. Spin trapping. Acc. Chem. Res. 4, 31-40 (1971).

2 Sciannamea, V., Jérome, R. \& Detrembleur, C. In-situ nitroxide-mediated radical polymerization (NMP) processes: their understanding and optimization. Chem. Rev. 108, 1104-1126 (2008).

3 Alberti, A., Benaglia, M., Laus, M., Macciantelli, D. \& Sparnacci, K. Direct ESR detection of free radicals in the RAFT polymerization of styrene. Macromolecules 36 , 736-740 (2003).

4 Kajiwara, A. A combination of electron spin resonance spectroscopy/atom transfer radical polymerization (ESR/ATRP) techniques for fundamental investigation of radical polymerization of (meth)acrylates. Polymer 72, 253-263 (2015).

5 Hermosilla, L., Calle, P., Tiemblo, P., García, N., Garrido, L. \& Guzmán, J. Polymerization of methyl methacrylate with lithium triflate. A kinetic and structural study. Macromolecules 46, 5445-5454 (2013).

6 Navarro, R. E. \& Ogawa, T. Effect of diphenylbutadiyne on the free radical polymerization of vinyl monomers in solution. J. Polym. Sci. A: Polym. Chem. 27, 2143-2149 (1989).

7 Hwang, J. S. \& Ogawa, T. ESR studies on the interaction of propagating radicals with diacetylenes. Polym. Bull. 23, 239-245 (1990).

8 Canizal, G., Burillo, G., Muñoz, E., Gleason, R. \& Ogawa, T. The interaction of poly(methacrylate) radicals with diphenyldiacetylenes. J. Polym. Sci. A: Polym. Chem. 32, 3147-3151 (1994).

9 Beristain, M. F., Bucio, E., Burillo, G., Muñoz, E. \& Ogawa, T. Study on the interaction of diarylbutadiynes with free radicals: interaction with propagating radicals of some vinyl monomers. Polym. Bull. 43, 357-364 (1999).

10 Burillo, G., Beristain, M. F., Sanchez, E. \& Ogawa, T. Effect of aromatic diacetylenes on polyurethane degradation by gamma irradiation. Polym. Degrad. Stab. 98, 1988-1992 (2013).

11 Cataldo, F., Ravagnan, L., Cinquanta, E., Castelli, I. E., Manini, N., Onida, G. \& Milani, P. Synthesis, characterization, and modeling of naphthyl-terminated $\mathrm{sp}$ carbon chains: dinaphthylpolyynes. J. Phys. Chem. B 114, 14834-14841 (2010).

12 Chen, S. N., Wu, W. Y. \& Tsai, F. Y. Homocoupling reaction of terminal alkynes catalyzed by a reusable cationic 2,2'-bipyridyl palladium(II)/Cul system in water. Green Chem. 11, 269-274 (2009).

13 Jones, A. \& Joyner, C. T. Preparation of 4,4',5,5'-tetranitro-1,1'-binaphthyl. J. Chem. Eng. Data 18, 105-108 (1973).

14 Chang, T. \& Kahn, A. H. Electron Paramagnetic Resonance Intensity Standard: SRM-2601, Description and Use National Bureau of Standards, Special Publication National Bureau of Standards, 39, 260-59, (Washington, DC, 1978).

15 Georgieff, K. K. Relative inhibitory effect of various compounds on the rate of polymerization of methyl methacrylate. J. App. Polym. Sci. 9, 2009-2018 (1965).

16 Ando, T., Kato, M., Kamigaito, M. \& Sawamoto, M. Living radical polymerization of methyl methacrylate with ruthenium complex: formation of polymers with controlled molecular weights and very narrow distributions. Macromolecules 29, 1070-1072 (1996).

17 Ando, T., Kamigaito, M. \& Sawamoto, M. Design of initiators for living radical polymerization of methyl methacrylate mediated by ruthenium(II) complex. Tetrahedron 53, 15445-15457 (1997).

18 Zeynalov, E. B. \& Friedrich, J. F. Anti-radical activity of fullerenes and carbon nanotubes in reactions of radical polymerization and polymer thermal/thermo-oxidative degradation. Mater. Test. 49, 265-270 (2007).

19 Kymakis, E. \& Amaratunga, G. A. J. Carbon nanotubes as electron acceptors in polymeric photovoltaics. Rev. Adv. Mater. Sci. 10, 300-305 (2005).

20 Stewart, D. \& Imrie, C. T. Role of C60 in the free radical polymerisation of styrene. Chem. Commun. 1383-1384 (1996).

21 Seno, M., Fukunaga, H. \& Sato, T. Kinetic and ESR studies on radical polymerization of methyl methacrylate in the presence of fullerene. J. Polym. Sci. A: Polym. Chem. 36, 2905-2912 (1998).

22 Arsalani, N. \& Geckeler, K. E. Radical bulk polymerization of styrene in the presence of fullerene[60]. Fullerene Sci. Technol. 4, 897-912 (1996). 